Check for updates

Cite this: RSC Adv., 2019, 9, 15798

\title{
Systematic evaluation of hydrophobic deep- melting eutectics as alternative solvents for the extraction of organic solutes from aqueous solution
}

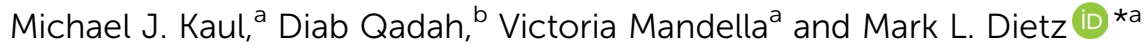

The partitioning of a number of organic compounds, including a series of $n$-alkanols and various simple, substituted benzene derivatives, between several hydrophobic (i.e., water-immiscible) deep eutectic solvents (HDESs) and water has been examined. The extent of extraction is shown to vary with the charge state of the molecule and the composition of the eutectic. In addition, the HDESwater distribution of a given solute is found to be directly proportional to (but typically less than) its partitioning in the octanol-water system, consistent with a significant role for solute hydrophobicity in the observed extraction behavior. Comparison of solute extraction into an HDES to that observed for other "unconventional" solvents (e.g., room-temperature ionic liquids and a soybean-derived oil) shows that hydrophobic deep eutectic solvents provide comparable or

superior extraction efficiency.
\end{abstract}

Received 3rd March 2019

Accepted 14th May 2019

DOI: $10.1039 / c 9 r a 01596 e$

rsc.li/rsc-advances

\section{Introduction}

For more than two decades, intense effort has been directed at the identification and characterization of alternatives to conventional molecular solvents. While once confined largely to studies of molten salts ${ }^{1,2}$ and supercritical fluids, ${ }^{3,4}$ these efforts have since expanded to encompass numerous types of ionic liquids (ILs), ${ }^{5-11}$ biomass-derived solvents (e.g. , glycerol, soybean oil methyl esters), ${ }^{11,12}$ liquid polymers, ${ }^{11,13}$ and deep eutectic solvents (DESs). ${ }^{11,14-16}$ Although eutectic mixtures have long been known, it is only with the introduction of environmentally benign DESs incorporating such components as urea and choline $\mathrm{e}^{17}$ that their enormous potential as alternative solvents has come to be widely appreciated. It is now recognized, for example, that the number of possible deep eutectic solvents may rival that of ionic liquids. Similarly, while concerns regarding the toxicity ${ }^{\mathbf{1 8 , 1 9}}$ and environmental impact ${ }^{19-23}$ of ionic liquids have increasingly been raised in recent years, deep eutectic solvents can be prepared from any number of materials whose "greenness" is unquestioned..$^{24,25}$ Finally, while the practical utility of ionic liquids is sometimes limited by their expense (fluorinated anions being particularly problematic in this context), ${ }^{26,27}$ numerous DESs can be prepared from inexpensive,

${ }^{a}$ Department of Chemistry and Biochemistry, University of Wisconsin-Milwaukee, Milwaukee, WI 53211, USA. E-mail: dietzm@uwm.edu; Fax: +1-414-229-5530; Tel: $+1-414-229-1748$

${ }^{b}$ Department of Chemistry, Birzeit University, Birzeit, P. O. Box 14, Ramallah, West Bank, Palestine commodity chemicals. ${ }^{28}$ These favorable characteristics have led to a range of applications in a variety of fields, including synthesis, ${ }^{29,30}$ catalysis, ${ }^{31}$ and chemical analysis, ${ }^{32}$ among others. While the vast majority of deep eutectic solvents described to date are hydrophilic, ${ }^{33,34}$ recent work has shown that a hydrophobic DES (designated hereafter as an HDES) can be readily obtained by combining menthol ${ }^{35-38}$ or an appropriate quaternary ammonium halide ${ }^{39-41}$ with a longchain carboxylic acid. The resultant liquids can then be employed, for example, as the basis of biphasic systems for the extraction of metal ions. ${ }^{42-44}$

Recently a number of reports demonstrating the extraction of various organic compounds (e.g., pesticides, pigments) into HDESs have appeared..$^{33,35,36,45-53}$ To date, however, little effort has been made to either elucidate the factors responsible for determining the efficiency of extraction of a given compound or to establish the utility of HDESs as media for extraction vis$\grave{a}$-vis other "unconventional" solvents. As a first step toward addressing these deficiencies, we have carried out a preliminary examination of the partitioning of a series of organic solutes, including various simple, substituted benzene derivatives, between selected HDESs and water. The results both illustrate the important role of solute hydrophobicity in determining the distribution of organic compounds in an HDES-water biphasic system and demonstrate that these systems can provide extraction efficiencies for organic solutes superior to both IL-water and bio-derived solvent-water systems. 


\section{Experimental}

\section{Reagents}

All deionized water was obtained from a Milli-Q2 system and exhibited a specific resistance of at least $18 \mathrm{M} \Omega \mathrm{cm}$. Methanol (99.8\%) and 1-pentanol ( $\geq 99 \%)$ were obtained from SigmaAldrich (Milwaukee, WI), as were tetraoctylammonium bromide (TOABr) (98\%), tetraoctylammonium chloride (TOACl) ( $\geq 97 \%$ ), menthol (99\%), and decanoic acid (98\%). All were employed without further purification. Concentrated hydrochloric acid (Optima ${ }^{\mathrm{TM}}$ grade), acetone (Optima ${ }^{\mathrm{TM}}$ grade), 1propanol (HPLC grade), 1-butanol (HPLC grade), and hexane (HPLC grade), were purchased from Fisher Scientific (Pittsburgh, PA), while standard $(0.100 \mathrm{~N})$ sodium hydroxide solution was obtained from Ricca Chemical Company (Batesville, IN). Ethanol ( $\geq 99.5 \%$ ) was purchased from Decon Labs Inc. (King of Prussia, PA). For liquid scintillation counting (LSC), Ultima Gold $^{\mathrm{TM}}$ (Perkin Elmer Corp., Waltham, MA) LS cocktail was employed.

With the exception of phenol (Moravek Biochemicals, Brea, CA) and toluene (American Radiolabeled Chemicals, St. Louis, $\mathrm{MO}$ ), all ${ }^{14} \mathrm{C}$-labeled reagents (aniline, benzoic acid, chlorobenzene, phthalic acid, $p$-toluic acid, and salicylic acid) were obtained from Sigma-Aldrich.

\section{Methods}

Preparation and characterization of deep eutectic solvents. To a $20 \mathrm{~mL}$ glass scintillation vial were added weighed quantities of appropriate hydrogen bond donor (HBD) and acceptor (HBA) components (e.g., decanoic acid and menthol, respectively) to provide a eutectic mixture with the desired (e.g., $1: 1$ ) HBD : HBA molar ratio. A stir bar was added, and the vial was placed into an oil bath held at a temperature just above the melting point of the higher-melting solid. The mixture was heated and gently stirred for $45 \mathrm{~min}$ to ensure thorough mixing and complete formation of the HDES. Upon removal from the bath, the sample was allowed to cool to room temperature and then to stand undisturbed for 24 hours. To verify formation of the eutectic, samples (5-10 mg) were analyzed by DSC on a TA Instruments Q20 Series differential scanning calorimeter.

Radiometric determination of organic solute partitioning. Prior to use in a partitioning experiment, a measured volume of the HDES of interest ( $c a .1000 \mu \mathrm{L}$ ) was combined with twice its volume of deionized water. The HDES-water mixture was vigorously agitated using a vortex mixer for several minutes and then allowed to stand for 24 hours. After centrifugation (3000 rpm for $30 \mathrm{~min}$ ), the HDES was carefully removed for use in partitioning measurements.

For these measurements, all of which were carried out at ambient temperature $\left(23 \pm 2{ }^{\circ} \mathrm{C}\right)$, equal volumes $(800-1000 \mu \mathrm{L})$ of the preconditioned HDES and water were combined. For ionizable solutes, aqueous phases consisting of water adjusted to $\mathrm{pH} 1.05$ or $\mathrm{pH} 11.00$ by addition of $\mathrm{HCl}$ or $\mathrm{NaOH}$ solution, respectively, were also utilized. In all cases, the volumes employed were chosen to facilitate sample handling, while minimizing radioactive waste generation. A known amount of the ${ }^{14} \mathrm{C}$-labeled organic analyte $(\sim 1 \mu \mathrm{L}$ of either the neat analyte (liquids) or a solution of the analyte in methanol (solids)) was then added and the mixture vortexed for $3 \mathrm{~min}$. Following $30 \mathrm{~min}$ of equilibration, the sample was centrifuged (4000 rpm for $50 \mathrm{~min}$ ) to achieve complete phase separation. The organic and aqueous layers were carefully separated and transferred to shell vials. Three $150 \mu \mathrm{L}$ aliquots of each phase were then transferred to scintillation vials containing Ultima Gold ${ }^{\mathrm{TM}}$ (6 $\mathrm{mL}$ ) for LS counting on a Perkin Elmer Tri-Carb 2810-TR Scintillation Analyzer. Blank samples comprising un-spiked $150 \mu \mathrm{L}$ aliquots of the HDES or aqueous phase alone were also counted. For each solute, the average of triplicate determinations of the blank-corrected count rates for the two phases were used to calculate the distribution ratio $(D)$, defined as follows:

$$
D_{\text {solute }}=\mathrm{CPM}_{\text {org }} / \mathrm{CPM}_{\mathrm{aq}}
$$

where $\mathrm{CPM}_{\text {org }}$ and $\mathrm{CPM}_{\mathrm{aq}}$ are the equilibrium concentrations of the solute in the organic (i.e., HDES) and aqueous phases, as represented by the respective blank-corrected count rates of the two phases. In all cases, the mass balance was determined to be satisfactory $( \pm 5-10 \%)$, thereby confirming the validity of the measured $D_{\text {solute }}$ values. The uncertainty associated with the values of $D_{\text {solute }}$ was found to be within $\pm 5 \%$.

Gas chromatographic determination of organic solute partitioning. As was the case for radiometric measurements of solute partitioning, the HDES of interest was first preconditioned by contacting a measured aliquot (ca. $1 \mathrm{~mL})$ with twice its volume of deionized water. Equal volumes $(\leq 1 \mathrm{~mL})$ of the pre-conditioned HDES and deionized water (the precise volumes again chosen to facilitate sample handling and subsequent analysis) were then combined, and a $50 \mu \mathrm{L}$ spike of the solute of interest ( $n$-alkanols, with $n=1-5$ ) was added. The HDES-water mixture was vigorously agitated using a vortex mixer for several minutes and then allowed to stand to achieve complete phase separation. After centrifugation (3000 rpm for $30 \mathrm{~min}$ ), the two phases were carefully separated and transferred to pre-cleaned glass GC vials for analysis. All measurements were performed on a HP-6890 series gas chromatograph (Agilent Technologies, Santa Clara, CA) equipped with a flame ionization detector (FID) and a $30 \mathrm{~m} \times 0.32 \mathrm{~mm}$ ID $\times 0.50 \mu \mathrm{m}$ df column (HP-INNOWAX Polyethylene Glycol). A column flowrate of $3.0 \mathrm{~mL} \mathrm{~min}^{-1}$, an injector temperature of $220^{\circ} \mathrm{C}$, and a $1: 10$ split ratio were employed. A detector temperature of $300{ }^{\circ} \mathrm{C}$ was maintained throughout, along with hydrogen, air,

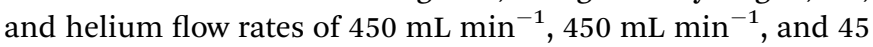
$\mathrm{mL} \min ^{-1}$, respectively. The initial GC oven temperature was set at $50{ }^{\circ} \mathrm{C}$ (hold time $=0 \mathrm{~min}$ ). From there, the temperature was gradually raised, first to $70{ }^{\circ} \mathrm{C}$ at a rate of $5{ }^{\circ} \mathrm{C} \mathrm{min}$ mold time $^{-1}$ (hold $=0 \mathrm{~min}$ ) and then to $220{ }^{\circ} \mathrm{C}$ at $75{ }^{\circ} \mathrm{C} \mathrm{min}{ }^{-1}$. For each solute, the solute concentration present in the two phases was used to calculate its distribution ratio $(D)$, defined as follows:

$$
D_{\text {solute }}=[\text { solute }]_{\text {org }} /[\text { solute }]_{\text {aq }}
$$

where $[\text { solute }]_{\text {org }}$ and [solute $]_{\mathrm{aq}}$ are the equilibrium concentrations of the solute in the organic (i.e., HDES) and aqueous 
phases, respectively. All measurements were again carried out in triplicate, and the mass balance was again found to be satisfactory in all instances. The uncertainty associated with the measured values of $D_{\text {solute }}$ is $c a . \pm 5 \%$.

\section{Results and discussion}

\section{HDES-water vs. octanol-water partitioning of simple organic solutes}

The biphasic distribution of organic solutes is most often described in terms of the octanol-water partitioning behavior of the compounds. Although alternative approaches have been developed (e.g., alkane-water partitioning ${ }^{54}$ ), the determination of octanol-water partition coefficients $\left(P_{\text {ow }}\right)$, defined for a solute $\mathrm{S}$ as the ratio $[\mathrm{S}]_{\mathrm{org}} /[\mathrm{S}]_{\mathrm{aq}}$ at equilibrium, remains the most widely accepted means of characterizing solute distribution and assessing the relative hydrophobicity of organic compounds. ${ }^{55}$ In large part, the widespread acceptance of $P_{\mathrm{ow}}$ values in this application is the result of the similarity of the dielectric properties of 1-octanol to those of lipids. This resemblance has led to the use of $P_{\text {ow }}$ values to model the transport of pharmaceuticals and biomolecules across biological membranes, ${ }^{56}$ the environmental fate of organic chemicals, ${ }^{57}$ and the effectiveness of pesticides, ${ }^{58}$ among numerous other applications. Our studies of the extraction of organic solutes from water into HDESs therefore began with a comparison of their HDES-water distribution ratios to established octanol-water partition coefficients. As can be seen from Fig. 1, which plots the measured values for a menthol-decanoic acid HDES against previously reported $P_{\text {ow }}$ values for a series of simple organic compounds, ${ }^{55}$ the general trend observed for the two parameters is the same. That is, those compounds displaying higher hydrophobicity (as reflected in the value of $P_{\text {ow }}$ ) also exhibit high $D_{\text {HDES-water values, }}$ with the absolute values of the latter measuring, on average, $c a$. $0.4 \log$ unit lower. This observation, in addition to being of fundamental interest, is of obvious practical importance from the perspective of the development of separation and recovery schemes for organic solutes involving liquid-liquid extraction or related techniques. That is, it is the absolute magnitude of its partition coefficient that determines the efficiency with which a given solute is extracted. (For example, for a solute with a $P_{\mathrm{ow}}$ of $10, c a$. $90 \%$ of it will be extracted by a single equal volume contact of a solute-containing aqueous solution.) Thus, if $D_{\text {HDES-water }}$ values vary systematically with $P_{\text {ow }}$ values, prediction of the likely utility of a particular HDES in the extraction of a given solute becomes possible. (With this in mind, we note that the HDES-water distribution ratios recently reported ${ }^{33}$ for several common neonicotinoid pesticides - acetamiprid, imidacloprid, and thiamethoxam - follow the general trend defined by the simple organic solutes, as shown in Fig. 1.) Similarly, the selectivity of extraction of one solute $v s$. another into a given solvent is determined by the relative magnitude of their partition constants. Therefore, the existence of a simple relationship between $P_{\text {ow }}$ and $D_{\text {HDES-water }}$ suggests that the estimation of the extraction selectivity expected for a given eutectic mixture may also be feasible.

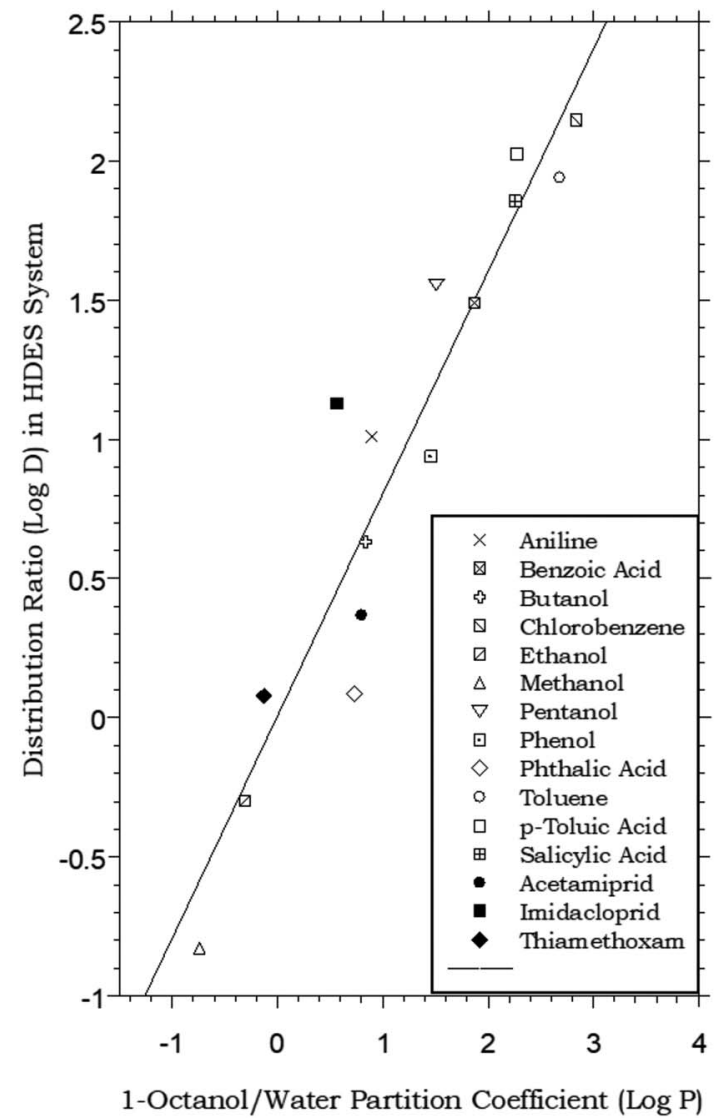

Fig. 1 Partitioning data for representative organic solutes in a biphasic HDES-water system vs. octanol-water. (HDES = menthol-decanoic acid; $1: 1$ molar ratio). The values for acetamiprid, imidacloprid, and thiamethoxam are taken from ref. 33. (Best fit line: $y=0.797 x+0.009$; $\left.R^{2}=0.8961\right)$.

Further insight into the partitioning process in the HDESwater system can be obtained by considering the $D_{\text {HDES-water }}$ values obtained for the series of $n$-alkanols. That is, from a semi$\log$ plot of $D_{\text {HDES-water }} v s$. the number of carbon atoms in the alkyl chain of the partitioning solutes $\left(n_{\mathrm{c}}\right)$, the free energy change associated with the transfer of a methylene unit from water into the HDES, $\Delta G_{\mathrm{CH}_{2}}$, can be readily calculated. Specifically, the free energy of transfer may be obtained from eqn (3):

$$
\Delta G_{\mathrm{CH}_{2}}=-2.303 R T E
$$

where $R$ is the universal gas constant (in kcal per K per mole), $T$ is the absolute temperature, and $E$ is the slope of the plot of $\log D_{\text {HDES-water }}$ vs. $n_{\mathrm{c}}$. Such a calculation here yields a free energy of transfer per methylene unit of $-0.77 \mathrm{kcal}$ per mole, a value identical to that reported for partitioning between water and alkanes (e.g., octane and dodecane) and essentially the same as that observed for the octanol-water system $(-0.79$ kcal per mole). ${ }^{59-61}$

\section{HDES-water vs. ionic liquid-water partitioning of simple organic solutes}

In that the values of $D_{\text {HDES-water }}$ apparently track those of $P_{\mathrm{ow}}$, the behavior of the HDES is reminiscent of that of the ionic liquid 
$\mathrm{C}_{4} \mathrm{mim}^{+} \mathrm{PF}_{6}{ }^{-}$, described previously, ${ }^{62,63}$ which provided distribution ratios for several of these same solutes that were proportional to, but somewhat less than, those observed in the octanolwater system. Thus, as shown in Fig. 2, the solute distribution ratios observed for the IL and the HDES follow the same general trend, although the latter values (in contrast to a recent report for several pesticides ${ }^{33}$ ) are typically greater. Solute partitioning in the HDES system parallels that observed previously with ILs in another important respect as well. That is, as noted previously for $\mathrm{C}_{4} \mathrm{mim}^{+} \mathrm{PF}_{6}{ }^{-}$, the partitioning of ionizable solutes between this IL and water exhibits a significant dependence on the aqueous phase $\mathrm{pH}$, with higher distribution ratios being observed under conditions where the neutral form of the solute predominates. ${ }^{62}$ As shown in Fig. 3, which depicts the $\mathrm{pH}$ variation in the distribution ratio of several representative organic solutes, the values

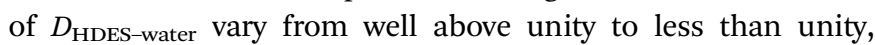
depending on the charge state of the molecule. For benzoic acid $\left(\mathrm{p} K_{\mathrm{a}}=4.19\right){ }^{62}$ for example, a more than 100 fold difference in extractability is observed between $\mathrm{pH} 1.05$ (neutral form predominates) and pH 11.00 (anionic form predominates), while a 1000 fold change in extractability of salicylic acid $\left(\mathrm{p} K_{\mathrm{a} 1}=2.87\right.$; $\left.\mathrm{p} K_{\mathrm{a} 2}=13.40\right)^{62}$ is seen under the same conditions. Both values are comparable to those observed for the IL-based extraction system, ${ }^{62}$ and clearly demonstrate that, despite a recent report suggesting that the ionized form of certain solutes may also be extracted into a DES, ${ }^{64}$ recovery of ionizable solutes extracted into

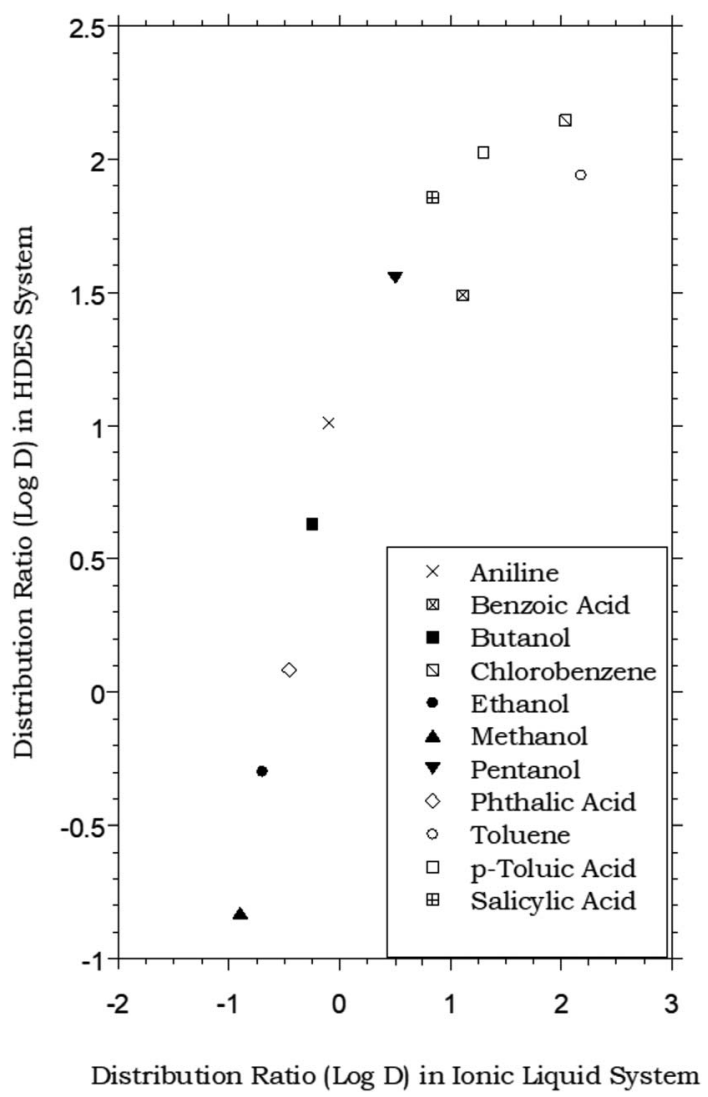

Fig. 2 Partitioning data for representative organic solutes in a biphasic HDES-water system vs. $\mathrm{C}_{4} \mathrm{mim}^{+} \mathrm{PF}_{6}{ }^{-}$(IL)-water. ${ }^{62}$ (HDES $=$ mentholdecanoic acid; 1 : 1 molar ratio).

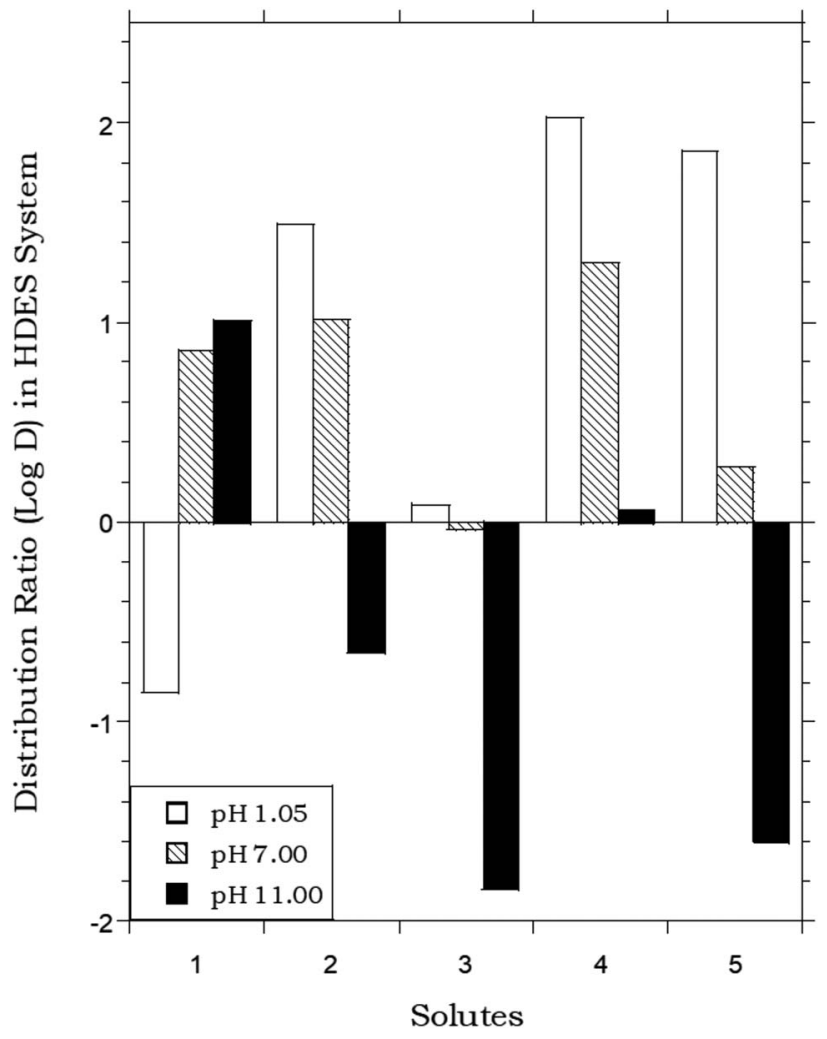

Fig. $3 D_{\text {HDES-water }}$ values for several ionizable organic solutes under acidic ( $\mathrm{pH} 1.05)$, neutral $(\mathrm{pH} 7.00)$, and basic $(\mathrm{pH} 11.00)$ conditions. (HDES $=$ menthol: decanoic acid; $1: 1$ mole ratio). Organic solutes: $1=$ aniline; 2 $=$ benzoic acid; $3=$ phthalic acid; $4=p$-toluic acid; $5=$ salicylic acid.

the menthol-decanoic acid HDES is easily achieved by means of a pH swing.

\section{HDES-water vs. SBME-water partitioning of simple organic solutes}

Like ILs, vegetable oils (e.g., olive oil) and related plant-derived carboxylic acid methyl esters (e.g., "biodiesel") have also been shown to provide satisfactory extraction of certain organic compounds from aqueous solution. ${ }^{\mathbf{1 1}}$ For the latter solvents, in particular, soybean methyl ester (SBME), the partitioning of a number of simple organic compounds has been shown to approximate the octanol-water partitioning behavior, although $D_{\text {SBME-water values are typically lower than analogous octanol-water }}$ values. ${ }^{65}$ As shown in Fig. 4, this means that at least for the limited range of solutes for which data are available, the menthol-based HDES and SBME are comparable in extraction behavior, following the same general trend. Of course, the variations in solvent composition achievable with HDESs far exceed those possible with natural oils or their esters, and in principle, such flexibility should provide an ability to "tune" extraction behavior in a way not readily achievable with the more conventional natural solvents.

\section{Effect of HDES composition on solute partitioning and extraction selectivity}

Indeed, as illustrated by the results presented in Table 1, which compares the values of $D_{\text {HDES-water }}$ observed for several organic 


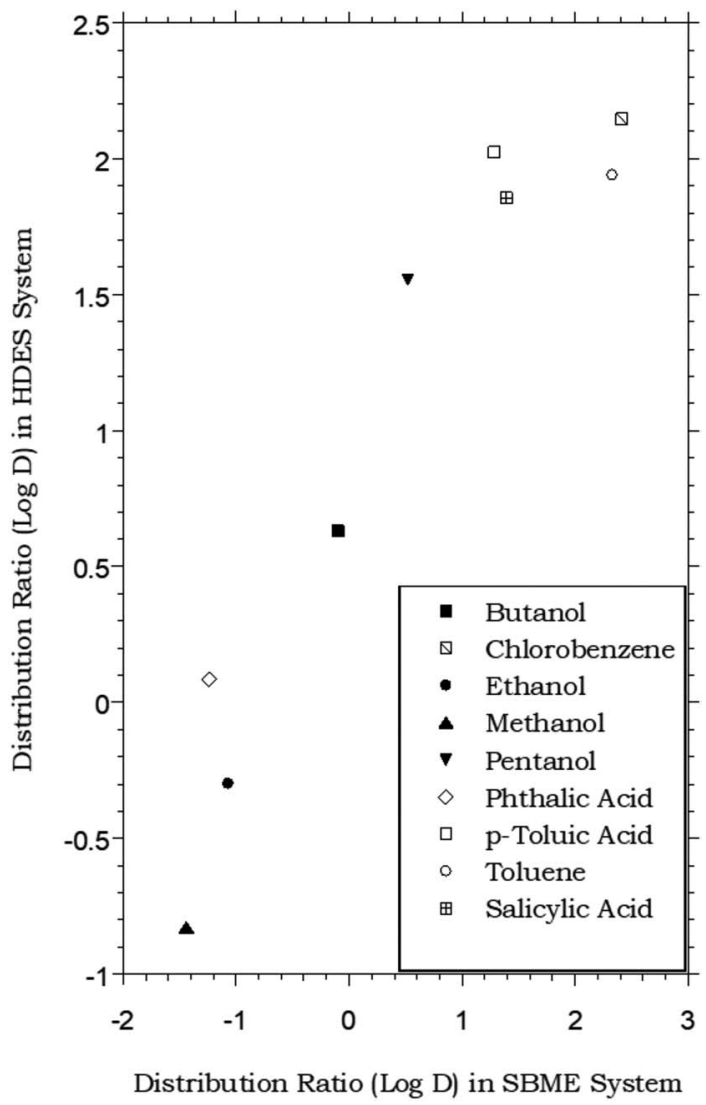

Fig. 4 Partitioning data for representative organic solutes in a biphasic HDES-water system vs. soybean methyl ester (SBME)-water. ${ }^{65}$ (HDES $=$ menthol-decanoic acid; $1: 1$ molar ratio).

solutes for the menthol-decanoic acid HDES to those obtained when either tetraoctylammonium bromide (TOABr) or the analogous chloride (TOACl) is employed as the hydrogen-bond acceptor, the observed solute distribution ratios do vary with the HDES composition. This observation is in agreement with a recent report describing the extraction of various bioactive

Table 1 Effect of eutectic composition ${ }^{a, b}$ on the partitioning of organic solutes

\begin{tabular}{lllll}
\hline & \multicolumn{3}{l}{$\log D_{\text {solvent-water }}$} & \\
\cline { 2 - 5 } Solute & 1-Octanol $^{c}$ & TOABr & TOACl & Menthol \\
\hline Aniline & 0.90 & 0.57 & 0.19 & 1.01 \\
Benzoic acid & 1.87 & 1.77 & 2.21 & 1.49 \\
Chlorobenzene & 2.84 & 2.55 & 2.70 & 2.15 \\
Phenol & 1.46 & 1.70 & 2.07 & 0.94 \\
Phthalic acid & 0.73 & 1.91 & 1.75 & 0.088 \\
Salicylic acid & 2.26 & 2.81 & 2.92 & 1.86 \\
Toluene & 2.68 & 1.74 & 1.68 & 1.94 \\
Toluic acid & 2.30 & 2.50 & 2.99 & 2.03
\end{tabular}

${ }^{a}$ Decanoic acid served as the HBD for all eutectics evaluated here. For the menthol-based HDES, the two components were present in a $1: 1$ molar ratio. For HDESs based on a quaternary ammonium salt, a $2: 1$ ratio of decanoic acid to the salt was employed. ${ }^{b}$ The menthol-, TOABr-, and TOACl-based HDESs were found to melt at $20.2{ }^{\circ} \mathrm{C}$, $7.67{ }^{\circ} \mathrm{C}$, and $-18.5{ }^{\circ} \mathrm{C}$, respectively. ${ }^{c}$ Ref. 54 . natural products by hydrophilic DESs, in which extraction yields were found to vary by as much as a factor of six, depending on the precise composition of the eutectic. ${ }^{64}$ If the relative extractability of a given pair of solutes in the various HDESs (i.e., the separation factor, $\alpha$ ) is considered, then there is again a clear difference between HDESs. For example, while the separation factor for toluene and phenol is only $1(0.04 \mathrm{log}$ units) in TOABr, it rises to more than 10 ( $1 \mathrm{log}$ unit) in the menthol-based HDES. Along these same lines, the chlorobenzene-toluene separation factor, which is $0.8 \log$ units in $\mathrm{TOABr}$, is only $0.2 \mathrm{log}$ units in the menthol HDES. While substantial additional work is clearly required to establish the precise relationship between HBA and HBD structure and the behavior of the resultant HDES as an extraction solvent, these initial results do indicate that significant tunability can reasonably be expected.

\section{Conclusions}

The results presented here represent the first systematic examination of the extraction of simple organic molecules into hydrophobic deep eutectic solvents. In addition to revealing the importance of solute hydrophobicity in determining the extent of partitioning between water and an HDES, they provide an indication of the utility of these solvents vis-à-vis established alternative solvents such as ionic liquids and bio-derived diluents. While preliminary, the results are encouraging, and suggest not only a straightforward relationship between partitioning in HDES-water and octanol-water systems, but also tunability on par with that seen for ionic liquids. Of course, considerable additional effort will be required to elucidate the underlying fundamentals of the partitioning process and to establish the relationship between the composition of a eutectic and its utility as an extraction solvent in various applications. Success in these efforts promises to vastly increase the range of extraction solvents available to the practitioners of separations. Work addressing these opportunities is now underway in this laboratory.

\section{Conflicts of interest}

There are no conflicts of interest to declare.

\section{Acknowledgements}

The authors gratefully acknowledge financial assistance provided by the Support for Undergraduate Research Fellows (SURF) Program of Office of Undergraduate Research of the University of Wisconsin-Milwaukee. In addition, the Chemical Sciences and Engineering (CSE) Division of Argonne National Laboratory is thanked for the generous gift of the ${ }^{14} \mathrm{C}$-labeled organic compounds employed in these investigations. Lastly, the authors thank Milwaukee Area Technical College (MATC) for access to the HP-6890 gas chromatograph. 


\section{References}

1 Molten Salts: From Fundamentals to Applications, NATO Science Series, II. Mathematics, Physics, and Chemistry, ed. M. Gaune-Escard, Kluwer Academic Publishers, Boston, 2002, vol. 52 .

2 R. M. Pagni, in Molten Salts and Ionic Liquids: Never the Twain?, ed. M. Gaune-Escard and K. R. Seddon, John Wiley, New York, 2010, pp. 279-299.

3 Supercritical Carbon Dioxide: Separations and Processes, ACS Symposium Series, ed. A. S. Gopalan, C. M. Wai, and H. K. Jacobs, American Chemical Society, Washington, DC, 2003, vol. 360 .

4 Green Chemistry using Liquid and Supercritical Carbon Dioxide, ed. J. M. DeSimone and W. Tumas, Oxford University Press, New York, 2003.

5 M. J. Earle and K. R. Seddon, Pure Appl. Chem., 2000, 72, 1391-1398.

6 N. Gathergood, M. T. Garcia and P. J. Scammells, Green Chem., 2004, 6, 166-175.

7 N. V. Plechkova and K. R. Seddon, Chem. Soc. Rev., 2008, 37, 123-150.

8 R. Giernoth, Angew. Chem., Int. Ed., 2010, 49, 2834-2839.

9 E. Santos, J. Albo and A. Irabien, RSC Adv., 2014, 4, 4000840018.

10 A. S. Amarasekara, Chem. Rev., 2016, 116, 6133-6183.

11 C. J. Clarke, W. C. Tu, O. Levers, A. Bröhl and J. P. Hallett, Chem. Rev., 2018, 118, 747-800.

12 Bio-Based Solvents, ed. J. François and R. Luque, John Wiley, Hoboken, NJ, 2017.

13 F. R. Mansour, L. Zhou and N. D. Danielson, Chromatographia, 2015, 78, 1427-1442.

14 Q. Zhang, K. De Oliveira Vigier, S. Royer and F. Jérôme, Chem. Soc. Rev., 2012, 41, 7108-7146.

15 E. L. Smith, A. P. Abbott and K. S. Ryder, Chem. Rev., 2014, 114, 11060-11082.

16 P. Liu, J. W. Hao, L. P. Mo and Z. H. Zhang, RSC Adv., 2015, 5, 48675-48704.

17 A. P. Abbott, G. Capper, D. L. Davies, R. K. Rasheed and V. Tambyrajah, Chem. Commun., 2003, 70-71.

18 D. Zhao, L. Liao and Z. Zhang, Clean: Soil, Air, Water, 2007, $35,42-48$.

19 T. P. T. Pham, C.-W. Cho and Y.-S. Yun, Water Res., 2010, 44, 352-372.

20 D. Coleman and N. Gathergood, Chem. Soc. Rev., 2010, 39, 600-637.

21 M. C. Bubalo, K. Radosevic, I. R. Redovnikovic, J. Halambek and V. G. Srcek, Ecotoxicol. Environ. Saf., 2014, 99, 1-12.

22 A. Jordan and N. Gathergood, Chem. Soc. Rev., 2015, 44, 8200-8237.

23 M. Made, J.-F. Liu and L. Pang, Environ. Sci. Technol., 2015, 49, 12611-12627.

24 Y. Dai, J. van Spronsen, G. J. Witkamp, R. Verpoorte and Y. H. Choi, Anal. Chim. Acta, 2013, 766, 61-68.
25 A. Paiva, R. Craveiro, I. Aroso, M. Martins, R. L. Reis and A. R. C. Duarte, ACS Sustainable Chem. Eng., 2014, 2, 10631071.

26 H. Yoon, G. H. Lane, Y. Shekibi, C. Howlett, M. Forsyth, A. S. Best and D. R. MacFarlane, Energy Environ. Sci., 2013, 6, 979-986.

27 A. Basile, H. Yoon, D. R. MacFarlane, M. Forsyth and P. C. Howlett, Electrochem. Commun., 2016, 71, 48-51.

28 B. Tang and K. H. Row, Monatsh. Chem., 2013, 144, 14271454.

29 M. Obst and B. Koenig, Eur. J. Org. Chem., 2018, 4213-4232.

30 L. S. Longo and M. V. Craveiro, J. Braz. Chem. Soc., 2018, 29, 1999-2025.

31 N. Guajardo, C. R. Muller, R. Schrebler, C. Carlesi and P. D. de Maria, Chemcatchem, 2016, 8, 1020-1027.

32 J. Werner, T. Grzeskowiak, A. Zgola-Grzeskowiak and E. Stanisz, TrAC, Trends Anal. Chem., 2018, 105, 121-136.

33 C. Florindo, L. C. Branco and I. M. Marrucho, Fluid Phase Equilib., 2017, 448, 135-142.

34 H. Passos, D. J. P. Tavares, A. M. Ferreira, M. G. Freire and J. A. P. Coutinho, ACS Sustainable Chem. Eng., 2016, 4, 2881-2886.

35 T. Kř́ižek, M. Bursová, R. Horsley, M. Kuchař, P. Tůma, R. Čabala and T. Hložek, J. Cleaner Prod., 2018, 193, 391-396.

36 R. Verma and T. Banerjee, Ind. Eng. Chem. Res., 2018, 57, 3371-3381.

37 B. D. Ribeiro, C. Florindo, L. C. Iff, M. A. Z. Coelho and I. M. Marrucho, ACS Sustainable Chem. Eng., 2015, 3, 24692477.

38 A. R. Zarei, M. Nedaei and S. A. Ghorbanian, J. Chromatogr. A, 2018, 1553, 32-42.

39 J.-J. Li, H. Xiao, X.-D. Tang and M. Zhou, Energy Fuels, 2016, 30, 5411-5418.

40 D. J. G. P. van Osch, L. F. Zubeir, A. van den Bruinhorst, M. A. A. Rocha and M. C. Kroon, Green Chem., 2015, 17, 4518-4521.

41 A. R. R. Teles, E. V. Capela, R. S. Carmo, J. A. Coutinho, A. J. Silvestre and M. G. Freire, Fluid Phase Equilib., 2017, 448, 15-21.

42 E. E. Tereshatov, M. Y. Boltoeva and C. M. Folden, Green Chem., 2016, 18, 4616-4622.

43 D. J. G. P. van Osch, D. Parmentier, C. H. J. T. Dietz, A. V. D. Bruinhorst, R. Tuinier and M. C. Kroon, Chem. Commun., 2016, 52, 11987-11990.

44 N. Schaeffer, M. A. R. Martins, C. M. S. S. Neves, S. P. Pinho and J. A. P. Coutinho, Chem. Commun., 2018, 54, 8104-8107.

45 D. Ge, Y. Zhang, Y. Dai and S. Yang, J. Sep. Sci., 2017, 41, 1635-1643.

46 R. Verma, M. Mohan, V. V. Goud and T. Banerjee, ACS Sustainable Chem. Eng., 2018, 6, 16920-16932.

47 D. J. G. P. van Osch, C. H. J. T. Dietz, J. van Spronsen, M. C. Kroon, F. Galluci, M. van Sint Annaland and R. Tuinier, ACS Sustainable Chem. Eng., 2019, 7, 2933-2942.

48 J. Cao, L. Chen, M. Li, F. Cao, L. Zhao and E. Su, Green Chem., 2018, 20, 1879-1886. 
49 C. Florindo, L. Romero, I. Rintoul, L. C. Branco and I. M. Marrucho, ACS Sustainable Chem. Eng., 2018, 6, 38883895.

50 M. A. Farajzadeh, M. R. A. Mogaddam and M. Aghanassab, Anal. Methods, 2016, 8, 2576-2583.

51 H. Wang, L. Hu, X. Liu, S. Yin, R. Lu, S. Zhang, W. Zhou and H. Gao, J. Chromatogr. A, 2017, 1516, 1-8.

52 J. Cao, M. Yang, F. Cao, J. Wang and E. Su, ACS Sustainable Chem. Eng., 2017, 5, 3270-3278.

53 S. Zhu, J. Zhou, H. Jia and H. Zhang, Food Chem., 2018, 243, 351-356.

54 M. H. Abraham, H. S. Chadha, G. S. Whiting and R. C. Mitchell, J. Pharm. Sci., 1994, 83, 1085-1100.

55 J. Sangster, J. Phys. Chem. Ref. Data, 1989, 18, 1111-1227.

56 F. Tsopelas, C. Giaginis and A. Tsantili-Kakoulidou, Expert Opin. Drug Discovery, 2017, 12, 885-896.

57 W. J. Doucette, Environ. Toxicol. Chem., 2003, 22, 1771-1778.
58 L. Shen and F. Wania, J. Chem. Eng. Data, 2005, 50, 742-768. 59 B. Y. Zaslavsky, L. M. Miheeva, N. N. Mestechkina and S. V. Rogozhin, J. Chromatogr., 1982, 253, 139-148.

60 B. Y. Zaslavsky, L. M. Miheeva and S. V. Rogozhin, J. Chromatogr., 1981, 212, 13-22.

61 B. Y. Zaslavsky, N. D. Gulaeva, E. A. Djafarov, E. A. Masimov and L. M. Miheeva, J. Colloid Interface Sci., 1990, 137, 147156.

62 J. G. Huddleston, H. D. Willauer, R. P. Swatloski, A. E. Visser and R. D. Rogers, Chem. Commun., 1998, 1765-1766.

63 J. G. Huddleston, A. E. Visser, W. M. Reichert, H. D. Willauer, G. A. Broker and R. D. Rogers, Green Chem., 2001, 3, 156-164.

64 L. Duan, L.-L. Dou, L. Guo, P. Li and E.-H. Liu, ACS Sustainable Chem. Eng., 2016, 4, 2405-2411.

65 S. K. Spear, S. T. Griffin, K. S. Granger, J. G. Huddleston and R. D. Rogers, Green Chem., 2007, 9, 1008-1015. 